\title{
Nivolumab Induced Acute Pancreatitis and Hyperbilirubinemia
}

\author{
Unnati Bhatia, $M D^{1 \#}$, Suryansh Bajaj, $M D^{2 \#}$, Darshan Gandhi, $M D^{3}$, Divyansh Bajaj, $M D^{4 *}$ \\ ${ }^{1}$ Department of Internal Medicine, Maulana Azad Medical College, New Delhi, India \\ ${ }^{2}$ Division of Neuroradiology, Yale School of Medicine, New Haven, CT, USA \\ ${ }^{3}$ Department of Diagnostic Radiology, University of Tennessee Health Science Center, Memphis, USA \\ ${ }^{4}$ Division of Pulmonary and Critical Care Medicine, Medical College of Wisconsin, Milwaukee, USA \\ ${ }^{\#}$ Equal Contribution
}

*Corresponding author: Divyansh Bajaj, MD, Division of Pulmonary Critical Care Medicine, Department of Medicine, Medical College of Wisconsin, 8701 W Watertown Plank Rd, Milwaukee, WI- 53226, USA, Tel: +1-(203)-610-4438, USA

\begin{abstract}
Immune checkpoint inhibitors (ICls) are a novel treatment modality that is quickly becoming the standard of care for the management of a multitude of neoplasms. While immunomodulation is a promising approach, it also carries a risk of immune-related adverse effects (irAEs). Nivolumab is an FDA-approved anti-programmed death-1 (anti-PD-1) checkpoint inhibitor. Nivolumab-induced, immune-related pancreatitis, and hyperbilirubinemia are rare but clinically significant examples of irAEs. Early recognition and prompt management of these conditions are critically important to ensure a better prognosis and prevention of subsequent complications.
\end{abstract}

\section{Keywords}

Immune checkpoint inhibitors, Adverse event, Drug-induced pancreatitis, Drug-induced hyperbilirubinemia, Nivolumab

\section{Introduction}

Cytotoxic T-cell lymphocyte-4 (CTLA-4) and programmed death-1 (PD-1)/ligand-1 (PD-L1) inhibitors have become a crucial part of the management of various malignancies. Due to their unique mechanism of action, these agents are called immune checkpoint inhibitors. It is estimated that $43.63 \%$ of all cancer patients in the US are eligible for treatment with $\mathrm{ICls}$, of which $12.46 \%$ of patients responded to therapy [1]. There is growing evidence that these agents confer a significant survival benefit in a wide spectrum of malignancies [2,3]. However, with increased use, an increase in the immune-mediated adverse effects of these agents referred to as irAEs, has also been seen. We report a unique case of acute pancreatitis and hyperbilirubinemia developing as an irAE of the use of Nivolumab immunotherapy.

\section{Case Presentation}

A 62-year-old man presented to the emergency department with severe abdominal pain for the past two days. He also reported several episodes of vomiting and nausea. Two months back, the patient was diagnosed with squamous cell carcinoma of the lung metastatic to his vertebrae and was commenced on Nivolumab immunotherapy a month back. Laboratory results were significant for white blood cells $12,500 /$ $\mu \mathrm{L}(3,300-8,600)$, amylase $232 \mathrm{U} / \mathrm{L}(42-130)$, lipase 279 $\mathrm{U} / \mathrm{L}(16-58)$ aspartate transaminase (AST) 64 units/L (13-44), alanine transaminase (ALT) 72 units/L (8-66), total bilirubin $6.4 \mathrm{mg} / \mathrm{dl}(0.2-1.2)$, direct bilirubin 5 $\mathrm{mg} / \mathrm{dl}(0.0-0.3 \mathrm{mg} / \mathrm{dl})$ and C-reactive protein $2.3 \mathrm{mg} /$ dl (0.00-0.16). Computed tomography (CT) scan of the abdomen demonstrated an enlarged, edematous pancreas with surrounding fluid and stranding (Figure 1). No gallstones or bile duct dilatation was noted. Due to high clinical suspicion, a hepatobiliary iminodiacetic acid (HIDA) scan was obtained, which came back normal. The patient had occasional alcohol intake. He was not taking any medications known to cause pancreatitis or hyperbilirubinemia except for Nivolumab. At this juncture, the patient was diagnosed with Nivolumabinduced pancreatitis and hyperbilirubinemia. Nivolumab 


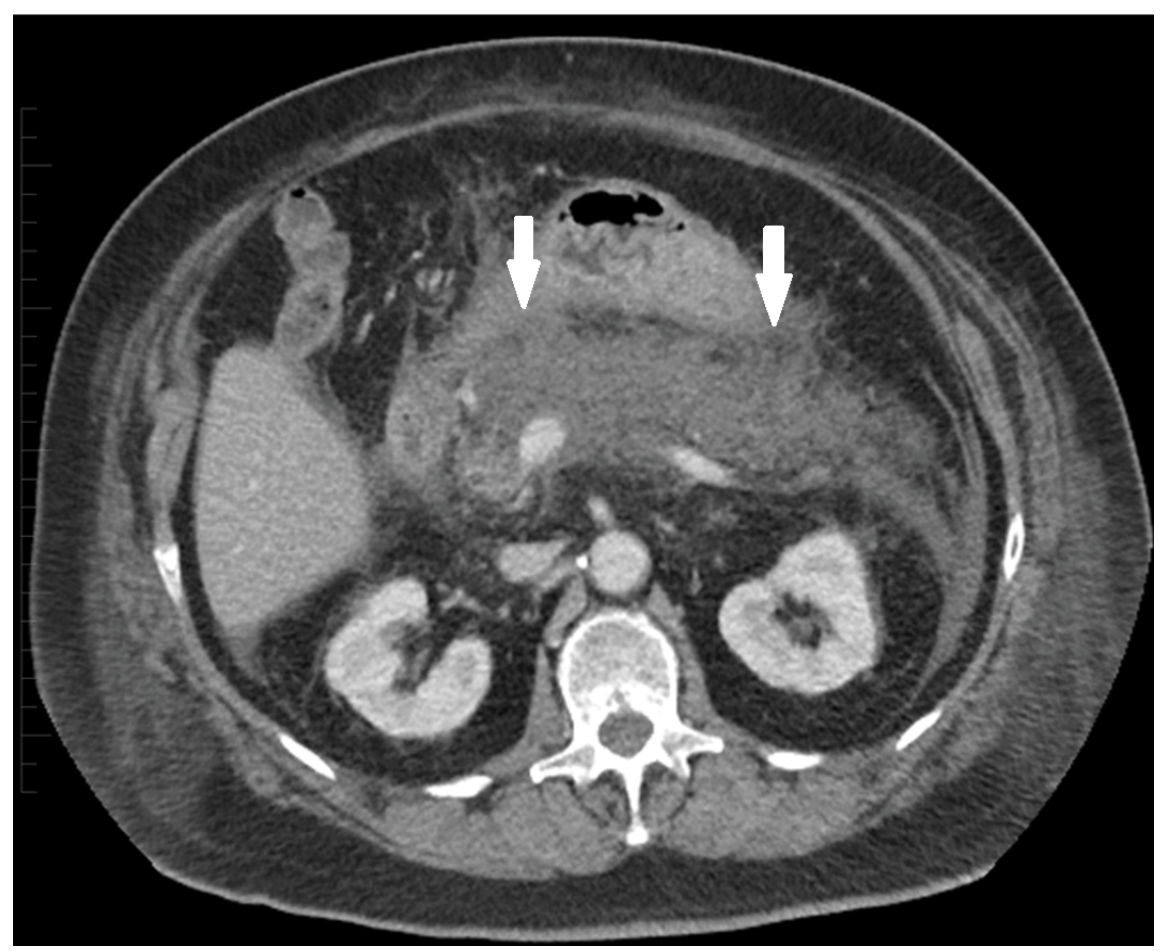

Figure 1: Axial CECT in a patient with abdominal pain demonstrates enlargement and edema of the pancreas with surrounding fluid and stranding, compatible with acute edematous pancreatitis. The predominant portion of the gland shows decreased intrinsic enhancement without definite necrosis.

therapy was discontinued. He was initially fluid resuscitated and was later started on high-dose steroid therapy with Prednisone at the $1 \mathrm{mg} / \mathrm{kg} /$ day dosing. The patient's symptoms significantly improved after 3 days of steroid treatment. He was prescribed a prolonged taper of oral Prednisone, tapered gradually over six weeks. On follow-up, the patient was asymptomatic and did not report any abdominal pain. Laboratory abnormalities had significantly improved with amylase $62 \mathrm{U} / \mathrm{L}$, lipase $78 \mathrm{U} / \mathrm{L}$, total bilirubin $1.8 \mathrm{mg} / \mathrm{dL}$, and direct bilirubin 0.6 $\mathrm{mg} / \mathrm{dL}$ on day 40 after treatment initiation.

\section{Discussion}

Suppression of T-cell mediated cytotoxicity is a well-known mechanism of oncogenesis, making immunomodulation with ICls a promising approach for the management of a wide array of cancers. AntiCTLA-4 and PD-1/PD-L1 blocking antibodies are the two commonly used classes of ICls that are currently FDAapproved for the management of several malignant conditions. Nivolumab, a human immunoglobulin G4 monoclonal antibody (IgG4), selectively inhibits the PD-1/PDL-1 interaction by binding to the PD-1 receptor on $T$ cells [4]. While this inhibition amasses a favorable anti-tumor immune response, it does not come without pitfalls. Firstly, the altered surface receptor interaction makes T-cells lose their ability to identify host cells. Secondly, while immune checkpoint inhibition has a stimulatory effect on effector T-cell proliferation, it also causes depletion of regulatory $\mathrm{T}$-cells, removing a critical anti-inflammatory mechanism of the immune system [5]. Together these factors may cause infiltration of immune cells into normal tissues, significantly increasing the risk of irAEs. While skin and the gastrointestinal system are the most commonly affected, almost every organ in the body may be subject to these adverse events $[6,7]$.

Immune-related pancreatic injury secondary to Nivolumab is a rare but important irAE with presentations ranging from an asymptomatic elevation in lipase levels to varying grades of clinically significant pancreatitis [7]. Immune-related pancreatitis may have a subacute, non-characteristic course and could be an underdiagnosed condition in patients with grade $3 / 4$ elevation in amylase and lipase levels post Nivolumab therapy. A recent meta-analysis studied the incidence of drug-induced pancreatitis associated with ICls. The study found an incidence of $2.7 \%$ for asymptomatic lipase elevation and $1.9 \%$ for grade 2 pancreatitis. A sub-group analysis to compare the incidence of druginduced pancreatitis between the two classes of ICls showed that therapy with anti-CTLA-4 agents was associated with a significantly higher incidence of pancreatitis (3.98\%, 95\% Cl: 2.92-5.05) as compared to anti-PD-1 agents (0.94\%, 95\% Cl: 0.48-1.40; $p<0.05)$ [8]. Characteristic symptoms and laboratory abnormalities in patients on Nivolumab should alert physicians to the possibility of this complication as early recognition and management can reduce associated morbidity and mortality. Nivolumab-induced pancreatitis largely remains a diagnosis of exclusion. A careful history and an abdominal ultrasound [9] can rule out common etiologies of pancreatitis, including alcohol use and gall stones. A temporal association of lab anomalies or symptoms with drug exposure may also help establish a 
causal relation. Previously reported cases have shown the onset of pancreatitis within 2-16 weeks of initiation of the drug [7].

Drug-induced hyperbilirubinemia has been commonly reported with isoniazid [10], amphotericin [11,12], allopurinol [12], and fluvastatin [13]. Nivolumab therapy has been associated with both hepatocellular and cholestatic patterns of hyperbilirubinemia, with the former being more common [14]. Cells of the biliary tract have an increased expression of human leukocyte antigen class 1 and 2, lymphocyte function-associated antigen 3 , and intercellular adhesion molecule 1 , making them susceptible to immune attacks in the setting of enhanced T-cell activity [4]. Similar to immune-related pancreatic injury, involvement of the biliary tract can also present on a spectrum ranging from asymptomatic hyperbilirubinemia, as seen in our case, to concomitant hepatotoxicity and cholangitis.

Most patients with irAEs respond to steroids $[4,15]$ with previously reported cases of immunerelated pancreatitis favorably responding to $1-2 \mathrm{mg} /$ $\mathrm{kg} /$ day prednisolone [16]. Mycophenolate mofetil may be considered in steroid-resistant cases $[4,15]$. Infliximab, which has demonstrated efficacy in steroidresistant irAE of colitis and pneumonitis, may also be a therapeutic option [16], except for cases with significant liver injury. A few cases have also demonstrated success with anti-thymocyte globulins, which act by depleting CD4 lymphocytes [17].

Nivolumab-induced immune-related pancreatic injury and hyperbilirubinemia are rare but significant irAEs that should be on the differential in a patient presenting with suggestive symptoms and laboratory abnormalities. Prompt treatment with immunosuppression usually shows a favorable response and may prevent complications. Further understanding of the predictive role of genetics and biomarkers hold significant potential for reducing irAEs.

\section{Declarations}

\section{Conflicts}

The authors have no conflicts of interest to declare.

\section{Funding}

No financial and material support was taken.

\section{Ethical approval}

No IRB approval needed.

Informed consent was obtained from the patient during an outpatient visit and was again obtained telephonically.

No content of the paper has been presented or published previously.

\section{Author contributions}

Literature review was done by all authors. Suryansh Bajaj and Unnati Bhatia wrote the first draft of the manuscript. The manuscript was critically revised by Darshan Gandhi and Divyansh Bajaj. Figure acquisition was done by Darshan Gandhi. All authors approved the final version to be published, and agree to be accountable for all aspects of the work.

\section{References}

1. Haslam A, Prasad V (2019) Estimation of the Percentage of US Patients With Cancer Who Are Eligible for and Respond to Checkpoint Inhibitor Immunotherapy Drugs. JAMA Network Open 2: e192535.

2. Borghaei H, Paz-Ares L, Horn L, Spigel DR, Steins M, et al. (2015) Nivolumab versus Docetaxel in Advanced Nonsquamous Non-Small-Cell Lung Cancer. The New England Journal of Medicine 373: 1627-1639.

3. Eggermont AM, Chiarion-Sileni V, Grob JJ, Dummer R, Wolchok JD, et al. (2016) Prolonged Survival in Stage III Melanoma with Ipilimumab Adjuvant Therapy. N Engl J Med 375: 1845-1855.

4. Kawakami H, Tanizaki J, Tanaka K, Haratani K, Hayashi $\mathrm{H}$, et al. (2017) Imaging and clinicopathological features of nivolumab-related cholangitis in patients with non-small cell lung cancer. Invest New Drugs 35: 529-536.

5. Pardoll DM (2012) The blockade of immune checkpoints in cancer immunotherapy. Nat Rev Cancer 12: 252-264.

6. De Velasco G, Je Y, Bossé D, Awad MM, Ott PA, et al. (2017) Comprehensive Meta-analysis of Key ImmuneRelated Adverse Events from CTLA-4 and PD-1/PD-L1 Inhibitors in Cancer Patients. Cancer immunology research 5: 312-318.

7. Hofmann L, Forschner A, Loquai C, Goldinger SM, Zimmer $L$, et al. (2016) Cutaneous, gastrointestinal, hepatic, endocrine, and renal side-effects of anti-PD-1 therapy. Eur J Cancer 60: 190-209.

8. George J, Bajaj D, Sankaramangalam K, Woo Yoo J, Joshi NS, et al. (2019) Incidence of pancreatitis with the use of immune checkpoint inhibitors $(\mathrm{ICI})$ in advanced cancers: A systematic review and meta-analysis. Pancreatology: Official Journal of the International Association of Pancreatology 19: 587-594.

9. Gandhi D, Ojili V, Nepal P, Nagar A, Hernandez-Delima FJ, et al. (2020) A pictorial review of gall stones and its associated complications. Clinical Imaging 60: 228-236.

10. Metushi I, Uetrecht J, Phillips E (2016) Mechanism of isoniazid-induced hepatotoxicity: Then and now. British Journal of Clinical Pharmacology 81: 1030-1036.

11. Bajaj D, Thumma S, Agrawal A, Mattana J (2019) Amphotericin B-Induced Hyperbilirubinemia. American Journal of Therapeutics 26: e765-e766.

12. Al-Kawas FH, Seeff LB, Berendson RA, Zimmerman HJ, Ishak KG (1981) Allopurinol hepatotoxicity. Report of two cases and review of the literature. Annals of Internal Medicine 95: 588-590.

13. Hartleb M, Biernat L, Kochel A (2002) Drug-induced liver damage -- a three-year study of patients from one gastroenterological department. Medical science monitor. International Medical Journal of Experimental and Clinical Research 8: Cr292-296. 
14. Zen Y, Yeh MM (2018) Hepatotoxicity of immune checkpoint inhibitors: A histology study of seven cases in comparison with autoimmune hepatitis and idiosyncratic drug-induced liver injury. Mod Pathol 31: 965-973.

15. Cramer P, Bresalier RS (2017) Gastrointestinal and Hepatic Complications of Immune Checkpoint Inhibitors. Current Gastroenterology Reports 19: 3.
16. Ikeuchi K, Okuma Y, Tabata T (2016) Immune-related pancreatitis secondary to nivolumab in a patient with recurrent lung adenocarcinoma: A case report. Lung Cancer 99: 148-150.

17. Wang W, Lie P, Guo M, He J (2017) Risk of hepatotoxicity in cancer patients treated with immune checkpoint inhibitors: A systematic review and meta-analysis of published data. International Journal of Cancer 141: 1018-1028. 